\title{
APS: percepción y nivel de participación en actividades de la Unidad de Salud Familiar, San Alfonso - Asunción, año 2010
}

\section{APS: perception and level of participation in activities of the Family Health Unit, San AlfonSo - Asunción, year 2010}

\author{
Romilio González Chamorro ${ }^{a}$
}

\begin{abstract}
Resumen
La Atención Primaria a la Salud (APS) fue implementada en Paraguay desde el año 2008. El presente estudio se propuso describir la percepción y el nivel de participación de la comunidad del barrio San Alfonso en las actividades y servicios de la Unidad de Salud Familiar en el marco de la APS durante el año 2010. La investigación fue descriptiva, con enfoque cuantitativo. Se realizó un muestreo no probabilístico por conveniencia en una población compuesta por 433 personas mayores de un total de 834 viviendas. Los resultados mostraron una población mayoritariamente femenina (76\%) de entre 41 a 60 años, que realizaban trabajos en el hogar $79 \%$ y se declararon jefas de hogar un $63 \%$. El $44 \%$ de todas las personas encuestadas culminó la secundaria, 34\% la primaría y $15 \%$ se encontraba en el nivel terciario. $61 \%$ se desempeñaba como ama de casa, $9 \%$ trabajadora doméstica fuera del hogar, $9 \%$ trabajadora independiente, $5 \%$ trabajo dependiente, 8\% jubilado/a y 8\% estudiante. El 6o\% participaba de los servicios médicos básicos de salud como la atención médica (43\%) y vacunaciones (29\%). 19\% asistió a charlas informativas de concientización y prevención de enfermedades. La mayoría conoce los Aty Guazú (reuniones informativas) de la APS pero el $84 \%$ dijo no participar porque les llega tarde la invitación. $44 \%$ de los que participan son de alguna organización como comisiones vecinales y grupos eclesiales un $35 \%$ respectivamente. La implementación del nuevo paradigma de la salud preventiva en la atención como política pública trajo consigo cambios en el modelo de atención así como grandes desafíos para toda la sociedad. Para sostener estos desafíos es necesario un consenso social y político del significado de participación comunitaria en la salud, así como considerar la percepción de la población para mejorar la atención y la comunicación entre el Equipo de Salud Familiar y la comunidad.
\end{abstract}

Palabras clave: APS, cuestión social, participación comunitaria.
Kera Yvoty: reflexiones sobre la cuestión social. Vol. 1, 2016, 103-110.

ISSN (impreso): 2519-7797

a Universidad Nacional de Asunción, Instituto de Trabajo Social, Paraguay.

Correspondencia a: romiliogch@gmail.com

Cita:

González Chamorro, R. (2016). APS: percepción y nivel de participación en actividades de la Unidad de Salud Familiar, San Alfonso - Asunción, año 2010. Kera Yvoty: reflexiones sobre la cuestión social, 1, 103-110.

Recibido:

1 noviembre 2016 Aceptado:

18 Diciembre 2016 


\begin{abstract}
Abstrac
Primary health care (PHC) was implemented in Paraguay since 2008. The present study aimed to describe the perception and level of participation of the community of the neighborhood of San Alfonso in the activities and services of the Family Health Unit in The PHC framework during 2010. The research was descriptive, with a quantitative approach. A non-probabilistic sampling was carried out for convenience in a population composed of 433 elderly people out of a total of 834 dwellings. The results showed a predominantly female population $(76 \%)$ between 41 and 60 years old, who performed work at home $79 \%$ and declared themselves to be heads of household $63 \% .44 \%$ of all the people surveyed completed secondary school, $34 \%$ of the primary school and $15 \%$ were in the tertiary level. $61 \%$ worked as housewife, $9 \%$ domestic worker outside the home, $9 \%$ self-employed, $5 \%$ selfemployed, $8 \%$ retired and $8 \%$ student. $60 \%$ participated in basic medical services such as health care $(43 \%)$ and vaccinations (29\%). 19\% attended informative talks on awareness and prevention of diseases. Most of them know the Aty Guazú (informative meetings) of the APS, but $84 \%$ said they did not participate because they are late for the invitation. $44 \%$ of those who participate are from some organization such as neighborhood commissions and church groups $35 \%$ respectively. The implementation of the new paradigm of preventive health care as a public policy brought about changes in the model of care as well as great challenges for the whole society. To sustain these challenges requires a social and political consensus on the meaning of community participation in health, as well as consider the perception of the population to improve care and communication between the Family Health Team and the community.
\end{abstract}

Keywords: PHC, social issue, community participation.

\section{Introducción}

En Paraguay, desde el 2008 se ha iniciado un proceso de cambio del modelo de atención de la salud basado en la Atención Primaria de la Salud APS- a través de las Unidades de Salud de la Familia -USF- en territorios sociales definidos como estrategia principal, según el contexto y particularidades de cada comunidad, para incrementar el acceso universal a servicios de salud.

Con la presente investigación se pretende ampliar el análisis de los resultados de la encuesta comunitaria aplicada en octubre del año 2010 por el Equipo de Práctica Pre-Profesional de estudiantes del 4to Curso de la Carrera de Trabajo Social de la Universidad Nacional de Asunción y el Equipo de Salud Familiar -ESF- de la USF San Alfonso del barrio Tacumbú desde un marco teórico elaborado para interpretar los resultados de la encuesta sobre percepción de la población sobre la APS y la participación comunitaria, así como para rescatar la experiencia y los aportes que la Práctica Pre-Profesional brinda al estudiantado y la sociedad.

El operativo de encuesta fue organizado y aplicado en el marco del proceso de inserción e intervención de la Práctica Pre-Profesional IV que el mencionado grupo de estudiantes realizó en el 2010 en la USF San Alfonso de Asunción a través de la Fundación Kuña Aty y el convenio que ésta poseía con el Ministerio de Salud Pública y Bienestar Social -M.S.P. y B.S.- para apoyar la gestión de las Agentes Comunitarias de la Salud -ACS- de las ESF.

En aquel momento, la dimensión de la participación, era algo nuevo tanto para todo el ESF como para la comunidad, por lo que fue necesario trabajar "en primer lugar la dimensión de participación comunitaria con los usuarios" (Urunaga, González, Giménez \& Dure 2010, p. 9).

La presente investigación permitirá al ESF y los actores comunitarios contar 
con una línea de base para fortalecer los servicios y la participación comunitaria basada en la APS.

\section{Objetivo general}

Describir la percepción y el nivel de participación de la comunidad en las actividades y servicios de la Unidad de Salud Familiar del barrio San Alfonso en el marco de la Atención Primaria de Salud.

\subsection{Objetivos específicos}

-Analizar las características sociodemográficas y socio-económicas de la población censada en la zona de influencia de la USF San Alfonso

-Identificar los servicios y las actividades de la USF en las que la población participa, los temas de interés y las dificultades de participación en las mismas

-Describir la percepción de la población sobre la APS, los servicios y las actividades brindadas por la USF -Identificar las formas de organización comunitaria en las que participa la población.

\section{Marco teórico}

\subsection{Cuestión social}

Para explicar las desigualdades sociales, Alayón \& Molina (2007) afirman que es necesario comprender el proceso histórico de composición del orden burgués y la sociedad patriarcal, por lo que es necesario entender estas desigualdades sociales, según la autora, como cuestión social, que define como la "contradicción entre el proletariado y la burguesía" (Alayón \& Molina, 2007), que se expresa y manifiesta en el cotidiano de la vida social.

Ahora, vale decir también que no hay nueva cuestión social. El desarrollo de la vida social y económica de la sociedad se ha vuelto más compleja, así como también las expresiones de la cuestión social se han complejizado, como lo sostienen Iamamoto y Netto (citados en Alayón \& Molina, 2007); "no hay nueva cuestión social porque es la misma causalidad de la relación capitaltrabajo, con expresiones derivadas de una producción cada vez más socializada y una apropiación cada vez más privatizada" (p. 7), esta desigualdad en la distribución de lo producido y de la riqueza es una desigualdad de carácter estructural y forma parte de la estructura social y política del actual sistema.

La cuestión social estudiada con esta investigación se expresa con una población desarticulada organizativamente que vive en condiciones de vulnerabilidad, exclusión, desigualdad social y con baja protección social, asentada en la periferia de la Capital conocida como Bañados de Asunción, producto de las migraciones forzadas del campo hacia las ciudades.

Los pobladores de la comunidad San Alfonso se asentaron en dicho lugar hace más de 55 años, producto de las migraciones forzosas del campo hacia la ciudad como consecuencia de la "matriz productiva, extractivista y agroexportadora" (Rojas, 2014, p. 8) que empezó desde principios del siglo XX y que se ha expandido con la soja y la ganadería extensiva e intensiva en la actualidad, en estos poblados de conformación espontanea e informal, la iglesia es la única institución que le ha dado atención y reconocimiento permanente (Perrota \& Navarro, 2003).

\subsection{Política pública y Atención Primaria en la Salud - APS}

En el periodo estudiado por esta investigación, se implementaba un cambio de estrategia para la atención de la salud que consistió en el paso del modelo basado en la salud curativa al modelo de salud preventiva que se enmarcó en la política pública con base en la APS con presencia en las comunidades a través de las USF.

Para la implementación de este cambio en el modelo de atención basado en la APS, el M.S.P. y B. S. había diseñado e implementado la Política Pública para la Calidad de Vida y Salud con Equidad como 
parte de la Propuesta de Políticas Públicas para el Desarrollo Social - PPDS 2010-2020 del Gobierno Nacional, que se sostiene en cuatro principios fundamentales que son la universalidad, integralidad, equidad y participación ciudadana, esta última se profundiza con este estudio.

La APS es la estrategia que articula el Sistema Nacional de Salud, "es un sistema de condiciones sociales, económicas y sanitarias básicas en la que la participación de las instituciones públicas, ya sean del gobierno central como local, articulan una misma estrategia de desarrollo en colaboración con sociedad civil y comunidades" (Consultora Pytyvo, 2012, p. 8).

\subsection{Unidad de Salud Familiar - USF}

La APS se materializa en las comunidades a través de la USF, que con los ESF son responsables del acompañamiento de aproximadamente 3.500 personas de un determinado territorio social. Cada ESF está compuesto por; 1 médico/a, 1 licenciado/a en enfermería u obstetricia, 1 auxiliar de enfermería, 1 auxiliar administrativo y 5 ACS (M.S.P. y B.S., 2009, p. 14).

Las USF son unidades que se "constituyen en la puerta de entrada al Sistema Nacional de Salud y hacen parte de la estrategia de APS) que plantea un nuevo paradigma en el modelo de atención (Guillen, 2011, p. 13), que atiende las necesidades y problemas de salud de toda la población de la zona de influencia a través de un equipo de profesionales de la salud, y "apunta a reducir la exclusión social, aumentando la cobertura y el acceso a las redes de atención integral a la salud" (Ruotti, Sequera, Orué \& Lampert. 2011, p. 2).

Para que todas las personas tengan garantizadas el acceso permanente y sin barrera alguna, estas "unidades están ubicadas lo más cerca de las personas, en las comunidades y barrios donde vive la gente" (M.S.P. y B.S., 20o8, p. 14).

\subsection{Agentes Comunitarios de Salud - ACS}

Los y las ACS son los promotores de salud, preferentemente son técnicos o referente sociales de la misma comunidad, conocen de primera mano la realidad y necesidad de su entorno y tienen la aceptación de la comunidad para actuar directamente en él. Son el primer nexo entre el establecimiento de salud y la comunidad (Urunaga et al., 2010, p.5).

\subsection{Participación Comunitaria}

Al concepto de la participación se le atribuye varios términos para indicar la participación de las personas en los ámbitos público, político, nacional, regional y local, por ejemploalgunos hablan de participación ciudadana, otros de participación social y también de participación comunitaria.

Los niveles de participación, Kliksberg (2011) lo vincula con los niveles de desarrollo de las democracias, y que la participación en la salud es un derecho de la población con diversas dimensiones de la misma, por ejemplo en "Paraguay se la vincula con la descentralización, y actuando principalmente en el nivel local" (Kliksberg, 2011, p. 19). Este nivel de participación está estrechamentevinculadocon la construcción del sentido comunitario, el arraigo y la identidad sociocultural que las familias realizan en su proceso de convivencia y aprendizaje social (SAS-CECTEC, 2010).

La participación en la gestión pública puede garantizar la transparencia, buena administración y satisfacción de las necesidades de los usuarios, por eso para los fines de la siguiente investigación, por participación comunitaria en la salud se entenderá a la capacidad de los actores comunitarios para decisión en la gestión y el control conjunto de los recursos y los servicios de salud, en coincidencia con la definición que el M.S.P. y B.S (2009) hace referencia en "Políticas Públicas para la Calidad de Vida y Salud con Equidad".

En la Ley 1032/1996, que crea el Sistema Nacional de Salud, se contempla la participación social a través de los Consejos de Salud para "impulsar el Sistema Nacional de Salud, promover la coordinación 
interinstitucional, participar en la formulación de lineamientos de la política y estrategia nacional, regional y local de salud" (Guillén, 2011, p. 5).

\section{Materiales y métodos}

La investigación planteada es de tipo descriptivo, de finalidad básica, alcance temporal transversal, de una amplitud microsocial. La fuente de la información es primaria. La investigación es de carácter cuantitativo.

\subsection{Población}

Muestreo no probabilístico por conveniencia. La población del estudio está compuesta por 433 personas mayores de un total de 834 viviendas ubicadas en la comunidad San Alfonso del Barrio Tacumbú, en un territorio social de 51 manzanas, entre la calle Alberdi hasta Pa'i Pérez y de 21 a 24 Proyectadas, dentro de la zona de intervención del ESF de la USF San Alfonso, divididos en 5 microterritorios. La unidad de medición es persona mayor responsable por familia.

Se realizó una encuesta comunitaria por barrido, para la cual se aplicó un cuestionario, de preguntas cerradas y una sola pregunta abierta, elaborado conjuntamente entre los estudiantes y ESF, que se anexa.

La encuesta se aplicó en octubre de 2010, por un Equipo de Encuestadores de 8 parejas integradas por 5 ACS del ESF, 4 enfermeras, 1 Doctor de Familia, 1 Auxiliar de Enfermería, 1 Facilitadora de Kuña Aty y los 4 estudiantes de Trabajo Social - UNA: Mercedes Duré, Zulma Urunaga, Sonia Giménez y Romilio González Chamorro.

El procesamiento y análisis preliminar de los datos estuvo a cargo del Equipo de Práctica, se realizó de manera manual, se elaboró una base de datos, se utilizó el programa Excel 2007. El análisis se reelaboró y profundizó con el presente trabajo utilizando como base la tabulación elaborada por el Equipo de Práctica.

Los aspectos éticos fueron considerados al realizar aviso previo a la comunidad a través de volanteadas, antes de cada encuesta se explicó los objetivos y alcances de la encuesta, la participación fuevoluntaria, se registró nombre, apellido y dirección de cada participante. El consentimiento informado se realizó en forma verbal. El porcentaje de la no aceptación de participación ha sido muy baja.

\subsection{Recursos para la aplicación de Encuesta}

La presente investigación fue realizada con recursos propios del estudiante proponente. La encuesta fue financiada con fondos propios de la U.S.F. e inversión propia y voluntaria de las/el estudiantes de Trabajo Social, aporte de la Fundación Kuña Aty. El costo para la aplicación de la encuesta fue de Gs. 800.000.

\subsection{Dimensiones y Variables}

\begin{tabular}{|c|c|c|}
\hline Dimensión & Variable & Indicadores \\
\hline $\begin{array}{l}\text { Socio- } \\
\text { demográfica }\end{array}$ & $\begin{array}{l}\text { Característica } \\
\text { poblacional }\end{array}$ & $\begin{array}{l}\text { Cantidad } \\
\text { de hogares } \\
\text { encuestados, } \\
\text { miembros de } \\
\text { familia, sexo, } \\
\text { edades }\end{array}$ \\
\hline $\begin{array}{l}\text { Socio- } \\
\text { económica }\end{array}$ & $\begin{array}{l}\text { Rol en la } \\
\text { familia; } \\
\text { Formación y } \\
\text { ocupación }\end{array}$ & $\begin{array}{l}\text { Jefatura de hogar, } \\
\text { nivel de formación, } \\
\text { ocupación, tipo de } \\
\text { trabajo }\end{array}$ \\
\hline Salud & $\begin{array}{l}\text { Atención } \\
\text { Médica }\end{array}$ & Servicios utilizados \\
\hline $\begin{array}{l}\text { Socio- } \\
\text { Comunitaria }\end{array}$ & $\begin{array}{l}\text { Participación } \\
\text { Comunitaria y } \\
\text { salud }\end{array}$ & $\begin{array}{l}\text { Interés de } \\
\text { participación, } \\
\text { conoce sobre } \\
\text { participación } \\
\text { comunitaria, tipo } \\
\text { de Organización } \\
\text { en la que participa, } \\
\text { actividades de } \\
\text { la USF, nivel y } \\
\text { dificultad de } \\
\text { participación en } \\
\text { actividades de USF, } \\
\text { temas de Interés, } \\
\text { conoce la APS }\end{array}$ \\
\hline
\end{tabular}




\section{Resultados}

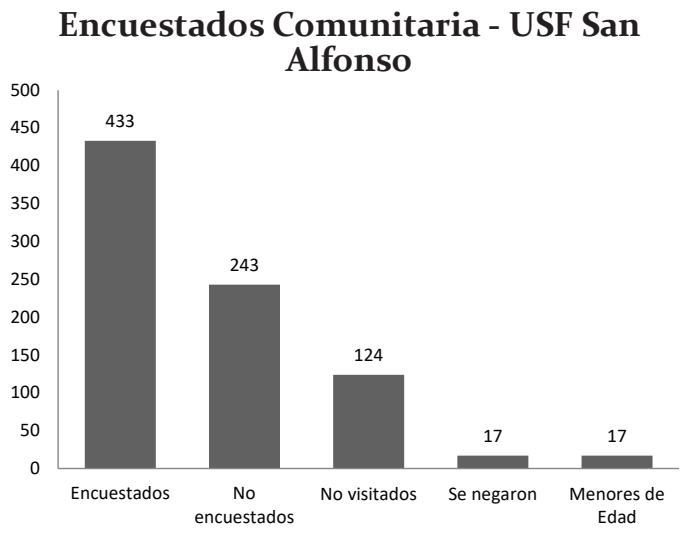

Figura 1. Población estudiada

De los 834 hogares de la comunidad, se logró encuestar a 433 personas mayores de edad, cada una responsable del hogar, En estos hogares viven aproximadamente 2034 personas.

No se pudo encuestar en 243 hogares visitados porque estaban cerrados, además no dio tiempo de llegar a 124 hogares debido al plazo fijado para la aplicación de la encuesta.

\subsection{Socio-demográficos y socios- económicos}

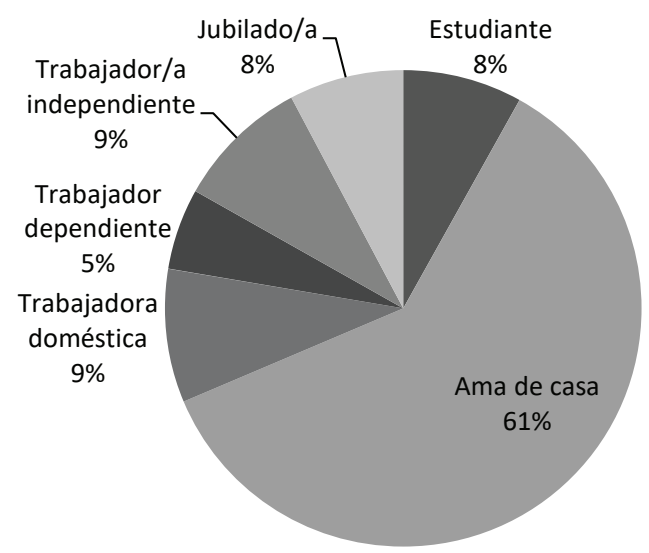

Figura 2. Ocupación Laboral
El $76 \%$ de las 433 personas encuestadas son mujeres de entre 41 a 60 años de edad (adultas medios), de las cuales un $63 \%$ se declara jefa de hogar. El $44 \%$ de todas las personas encuestadas culminaron la secundaria, 34\% la primaría y solo un $15 \%$ declaró estudiar en el nivel terciario.

Entre las ocupaciones: el $79 \%$ realiza trabajo en el hogar de las cuales $61 \%$ declaró ser ama decasa, el 9\% trabajadora domésticayotros $9 \%$ trabajadoras independientes (comerciantes en un $54 \%$, modista, vendedor/a ambulante y zapateros, carpinteros, peluqueras).

\subsection{Salud y socio-comunitaria}

El 6o\% participa o se vincula a través de los servicios médicos básicos de salud como la atención médica (43\%) y las las vacunaciones (29\%) y solo un $19 \%$ respondió que asistió a las charlas informativas, de concientización y prevención de enfermedades.

Las encuestadas tienen interés en temas clínico-médicos como la hipertensión (24\%), diabetes (22\%), nutrición y alimentación (18\%) y salud sexual y reproductiva (13\%).

\subsection{Organización}

La mayoría conoce los Aty Guazú (reuniones informativas) de la APS pero el $84 \%$ dijo no participar de ellas porque les llega tarde la invitación (64\%), tienen problemas de horario para asistir a las actividades de la USF (43\%), les coincide con el trabajo (23\%), motivos familiares (13\%), por enfermedad (6\%).

De los encuestados, el $44 \%$ de los que participan son de alguna organización como las comisiones vecinales y los grupos ecleciales de las iglesias en un 35\% respectivamente. La percepción sobre la APS y USF, la comunidad espera que el ESF capacite e informe más sobre la APS mejorando su atención y comunicación con la población usuaria. 


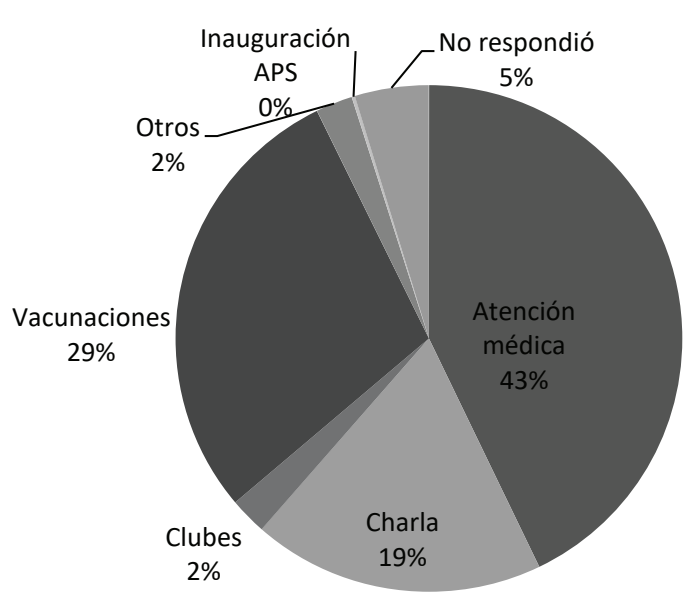

Figura 3. Participación en la USF

\section{Discusión}

La implementación del nuevo paradigma de la salud preventiva en la atención como política pública y con la incorporación de los principios de universalidad, integralidad, equidad y participación social trajo consigo cambios en el modelo de atención así como grandes desafíos para toda la sociedad.

Para sostener estos desafíos y cambios es necesario un consenso social y político del significado de participación comunitaria en la salud, así como la de considerar la

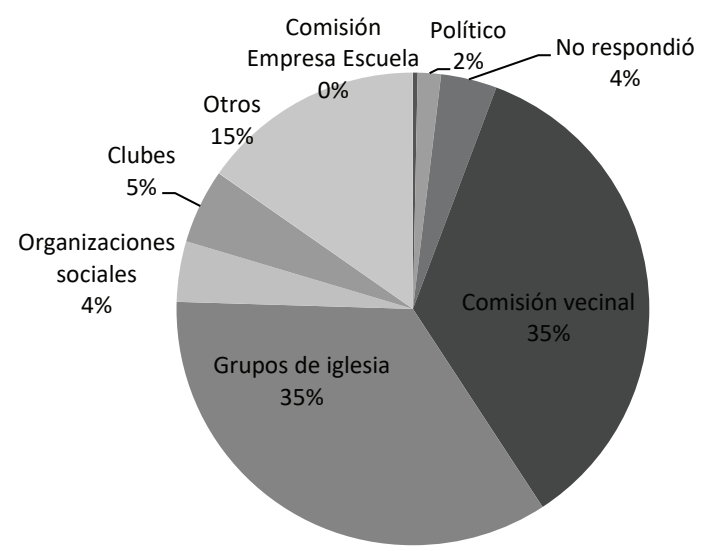

Figura 4. Organización percepción de la población para mejorar la atención y la comunicación entre el ESF y la comunidad, orientando y articulando la participación de las organizaciones ya existentes (comisiones vecinales y grupos eclesiales) a la estrategia de la APS, lo que implica, como recomienda la Consultora Pytyvo, una mayor capacitación de los profesionales en medicina comunitaria y de apostar por la APS como "herramienta de desarrollo local y nacional (...) en beneficio del desarrollo social" (Consultora Pytyvo, 2012, pp. 94-95).

\section{Conclusiones}

La población usuaria disponible durante el día en la comunidad de la USF San Alfonso es mayoritariamente femenina, realizan tareas en la casa, se asumen jefas de hogar.

Lapoblación encuestada conocesobre los Aty Guazú de la APS pero no participan de ellos, asisten más en actividades de la clínica médica y si bien tienen experiencias organizativas, éstas están desarticuladas y desvinculadas de la estrategia de la APS.

Es por eso que para lograr una mayor participación de los/as actores/as comunitarios en la misma, es necesario reforzar el rol de los/as profesionales del área social para profundizar los procesos por mayores conquistas sociales de cada sector, ampliar los derechos y asumir mayores compromisos que profundicen trasformaciones hacia una cultura preventiva de la salud.

\section{Referencias Bibliográficas}

Alayón, N. \& Molina, M. L. (2007). La desigual social: desarrollo y desafíos del Trabajo Social desde la reconceptualización en América Latina. Revista Textos y Contextos, 6(1), 34-68. Recuperado de http:// revistaseletronicas.pucrs.br/ojs/index. php/fass/article/viewFile/1044/3231

Consultora Pytyvo. (2012). Monitoreo del programa de Unidad de Salud de la Familia (USF), como programa 
emblemático de la política de desarrollo social, Paraguay para todos y todas 2010-2020. Recuperado de http:// www.mspbs.gov.py/planificacion/ wp-content/uploads/2012/o6/ DIPLANP-2012.pdf

Guillen, M. C. (2011). Paraguay: sistema de salud en Sudamérica: desafíos hacia la integralidad y equidad. Recuperado de http:// www.mspbs.gov.py/planificacion/ wp-content/uploads/2012/o7/SSSPARAGUAY-2011.pdf

Kliksberg, B. (2011). Estrategias y metodologías para promover la participación social en la definición e implantación de políticas públicas de combate a las inequidades en salud. Recuperado de http://cmdss2on. org/site/wp-content/uploads/2011/o7/ Participaci\%C3\%B3n-Social_BernardoKliksberg.pdf

Ministerio de Salud Pública y Bienestar Social \& Fundación Kuña Aty. (2008). Fortalecimiento de servicios de atención primaria, con énfasis en salud materno infantil y sexual reproductiva. Recuperado de http://www.aecid.org. py

Ministerio de Salud Pública y Bienestar Social. (2009). Políticas Públicas para la calidad de vida y salud con equidad. Asunción: M.S.P. y B.S.

Perrotta, M. \& Navarro, E. (2003). Documento sobre la experiencia vivida por dos Trabajadoras Sociales. Un camino hacia la libertad, salud comunitaria.

Presidencia de la República. Gabinete Social. (2009). Paraguay para todos y todas. Propuesta de políticas públicas para el desarrollo social 2010-2020. Asunción: Gabinete Social.

Rojas, L. (2014). La tierra en disputa. Extractivismo, exclusión y resistencia. Asunción. Base IS. Recuperadodehttp:// www.baseis.org.py/base/adjuntos/ Tierra $\% 2$ oy $\% 2$ oextractivismo $\% 20$ en\%2oParaguay\%2oBASE.pdf
Ruotti, M., Sequera, M., Orué, E. \& Lampert, N. (2011). Actualización de la línea de base 2008-2010 y aplicación de las encuestas año 2010: hábitos para la prevención de la diarrea, percepción de la calidad de la atención que brindan las Unidades de Salud Familiar (USF): informe final. Asunción: IICS. UNA. Recuperado de http://www.mspbs. gov.py/planificacion/wp-content/ uploads/2012/o6/Calidad_atencion_ USF_-Ruoti2011.pdf

Secretaría de Acción Social \& Centro de Educación, Capacitación y Tecnología Campesina. (2010). Cuaderno pedagógico, facilitadores/ as comunitarios: Primera etapa. Asunción: UNFPA. GTZ.

Urunaga, N., González, R., Giménez, S. \& Dure, M. (2010). Conocemos nuestra comunidad y construimos la participación comunitaria en la U.S.F. San Alfonso. Proyecto de Intervención para la U.S.F. San Alfonso del Barrio Tacumbú. Asunción: Cátedra de Práctica Pre-Profesional IV, Carrera de Trabajo Social, Universidad Nacional de Asunción.

USAID \& GEAM. (2010). Programa emblemático gubernamental. Unidades de salud familiar. Informe de evaluación de proceso. Recuperado de http:// www.mspbs.gov.py/planificacion/ wp-content/uploads/2012/o4/ APS_Programa_emblematico_ Caballerozo10.pdf 